\title{
Scattering of two coherent beams by multiple centers*
}

\author{
Ming Chiang Li \\ Department of Physics, Virginia Polytechnic Institute and State University, Blacksburg, Virginia 24061
}

(Received 1 April 1974; revised manuscript received 13 May 1974)

\begin{abstract}
This is a continuation in the theoretical study of scattering initiated by two coherent beams. Here the scattering by multiple independent centers is discussed. The presence of these centers involves some problems, which are not present in scattering with a single beam. I show how these problems may be overcome, thus allowing the phase of the scattering amplitude to be determined experimentally.
\end{abstract}

\section{INTRODUCTION}

In most cases, scattering experiments initiated by a single beam yield information only on the magnitude of the scattering amplitude but no information on the phase. Since the phase and magnitude are two independent parts of a scattering amplitude, such incomplete knowledge means that the scattering amplitude is only partially observed.

There are a limited number of exceptions to the above statement. A well known case is that of the Coulomb interference method. ${ }^{1}$ This method, however, is only useful when the interaction in the scattering process can be divided into two parts, one of which is the known Coulomb potential. Through the interference with the known Rutherford amplitude, the phase of the remaining part of the amplitude may be determined. The separation of the interaction into two parts does not'necessarily mean that the scattering amplitude can also be separated into two parts. Additional assumptions must be added, which reduce the effectiveness of the Coulomb interference method. Nonetheless, this method is used in proton-proton scattering ${ }^{2}$ for the determination of the phase of the strong nucleon scattering amplitude near the forward direction. It must be noted that this method is only valid when the exact Coulombic force is known or present in a given scattering problem.

The method of phase-shift analysis ${ }^{3}$ is another exception.' At sufficiently low energy, the cross section is isotropic and the $s$ wave dominates the scattering amplitude. The $s$-wave phase shift is then "continuously" followed by taking measurements at somewhat higher energies. As higher partial waves successively enter, they will be followed closely and detected from the measurements. The best known applications of this method are in phase-shift analyses in nucleon-nucleon and meson-nucleon interactions. ${ }^{3}$. Even these are not simple cases because the procedure depends upon arguments of continuity and other supplemental assumptions for the correlation of phase shifts at different energies. In some of these situations the procedure becomes ambiguous ${ }^{4}$ when there exists a number of different sets of phase shifts.

Despite the inevitable difficulties, one of the goals of quantum mechanics is to measure directly the phase of a scattering amplitude. One of the more remarkable attempts is the intensity-correlation method ${ }^{5}$ employed by Hanbury-Brown and Twiss. They showed, by using two rather intense and incoherent beams, that it is experimentally feasible to measure the phase of a scattering amplitude up to an angle-independent constant. This method utilizes the fluctuations in the incoherent beams. The interference effects associated with the fluctuations are detected with coincidence measurements at two different angles. Such an experiment has not been performed for quantum mechanical scattering as yet.

A more recent suggestion is the time-delay method $^{6}$ for direct phase determination. It requires reproducible wave packets of incident particles; however, the construction of such wave packets has not yet been achieved experimentally.

By far it seems to me the most promising approach for phase determinations is that of using direct interference experiments. Unfortunately, the very short de Broglie wavelength of most particle beams has discouraged people from seriously considering such possibilities. In a previous paper, ${ }^{7}$ I have discussed the fixed-potential scattering of two coherent beams. These are not impossible to obtain. There do exist coherent laser and electron beams. Coherent laser beams are well known. The coherent electron beams, ${ }^{8}$ although not so familiar, have been employed since the early 1950's. Unfortunately, the experimental techniques for obtaining the coherent electron beams are not receiving the attention they merit. ${ }^{9}$

In real scattering experiments the targets are macroscopic samples containing many independent centers. In this paper the scattering of two coherent beams by these centers is considered. In Sec. II, I lay down the theoretical foundation of the problem. It is different from that of singlebeam scattering in that the observed cross section of two coherent beams always depends on the spatial distribution of the centers. In Sec. III the 
centers are restricted to a plane surface. The phase of the scattering amplitude is easy to obtain in this case. For the three dimensions, if the centers are randomly distributed, the phase can be obtained through coincidence measurements. ${ }^{10}$ This is discussed in Sec. IV. In Sec. V, I deal with the crystal diffraction of two coherent beams. It is shown that the phase of the structure factor, which is the scattering amplitude of a unit cell in the crystal, can be measured in the diffraction experiment. The primary experiment ${ }^{11}$ of this kind was done more than 20 years ago.

\section{SCATTERING BY INDEPENDENT CENTERS}

In an idealized scattering experiment, a single fixed scattering center is bombarded by particles incident along fixed directions. The effect of the scattering center on the particles is represented by a potential energy which is appreciably different from zero only within a finite region. This is the starting point for quantum potential scattering problems. In a previous paper ${ }^{7}$ on scatterings initiated by two coherent beams, I also took the same view. In real scattering experiments, there are complications which arise from the fact that experimental targets are macroscopic samples containing many independent scattering centers. These complications do not usually create problems in single-beam scattering for which the number of the scattered particles detected is proportional to the number of independent scattering centers. However, for coherent-beam scatterings the problem is not quite so simple.

Let us assume that in the target region there are $N$ independent scattering centers, by which it is meant that the scattering from each of these centers is independent of the presence of the other centers. The position of each center is dencied by the vector $\vec{r}_{i}$. The coordinate system is so chosen that its origin is located at the mean position of scattering centers $\left(\sum_{i=1}^{N} \overrightarrow{\mathbf{r}}_{i}=0\right)$. This choice is just for convenience and does not impose any restriction. The two incident coherent beams have the momenta $\overrightarrow{\mathrm{k}}_{1}$ and $\overrightarrow{\mathrm{k}}_{2}$ such that

$$
\overrightarrow{\mathrm{k}}_{1}^{2}=\overrightarrow{\mathrm{k}}_{2}^{2}=k^{2}=2 m E / \hbar^{2},
$$

where $E$ is the energy of the beams and $m$ is the mass of the incident particles. The incident coherent beams are described by the wave function

$$
e^{i \overrightarrow{\mathrm{k}}_{1} \cdot \overrightarrow{\mathrm{r}}}+a e^{i \overrightarrow{\mathrm{k}}_{2} \cdot \overrightarrow{\mathrm{r}}},
$$

where $a$ denotes the relative phase and amplitude of these two waves. With respect to each center, the scattering is independent and the scattered wave function $\psi_{i}(\vec{r})$ satisfies the Schrödinger equation

$$
-\frac{\hbar^{2}}{2 m} \nabla^{2} \psi_{i}(\overrightarrow{\mathrm{r}})+V\left(\overrightarrow{\mathrm{r}}-\overrightarrow{\mathrm{r}}_{i}\right) \psi_{i}(\overrightarrow{\mathrm{r}})=E \psi_{i}(\overrightarrow{\mathrm{r}})
$$

and the asymptotic condition

$$
\psi_{i}(\overrightarrow{\mathrm{r}}) \underset{r \rightarrow \infty}{\longrightarrow} e^{i \overrightarrow{\mathrm{k}}_{1} \cdot \overrightarrow{\mathrm{r}}}+a e^{i \overrightarrow{\mathrm{k}}_{2} \cdot \overrightarrow{\mathrm{r}}}+F_{i}\left(\overrightarrow{\mathrm{k}}_{1}, \overrightarrow{\mathrm{k}}_{2} ; \overrightarrow{\mathrm{n}}\right) \frac{e^{i k r}}{r},
$$

where $V\left(\overrightarrow{\mathbf{r}}^{\prime}\right)$ is the potential, which is assumed to be the same for all the centers. The direction of the scattered wave has been denoted by $\vec{n}$. $F_{i}\left(\overrightarrow{\mathrm{k}}_{1}, \overrightarrow{\mathrm{k}}_{2} ; \overrightarrow{\mathrm{n}}\right)$ is the coherent scattering amplitude for the $i$ th center. To solve the problem, we make use of a new coordinate system with its origin located in the $i$ th center. Then Eqs. (2.3) and (2.4) have the new forms

$$
-\frac{\hbar^{2}}{2 m} \nabla^{2} \psi_{i}^{\prime}\left(\overrightarrow{\mathbf{r}}^{\prime}\right)+V\left(\overrightarrow{\mathbf{r}}^{\prime}\right) \psi_{i}^{\prime}\left(\overrightarrow{\mathrm{r}}^{\prime}\right)=E \psi_{i}^{\prime}\left(\overrightarrow{\mathrm{r}}^{\prime}\right)
$$

and

$$
\begin{aligned}
\psi_{i}^{\prime}\left(\overrightarrow{\mathrm{r}}^{\prime}\right) \equiv & \psi_{i}(\overrightarrow{\mathrm{r}}) \underset{r \rightarrow \infty}{\longrightarrow} e^{i \overrightarrow{\mathrm{k}}_{1} \cdot\left(\overrightarrow{\mathrm{r}}^{\prime}+\overrightarrow{\mathrm{r}}_{i}\right)}+a e^{i \overrightarrow{\mathrm{k}}_{2} \cdot\left(\overrightarrow{\mathrm{r}}^{\prime}+\overrightarrow{\mathrm{r}}_{i}\right)} \\
& +F_{i}\left(\overrightarrow{\mathrm{k}}_{1}, \overrightarrow{\mathrm{k}}_{2} ; \overrightarrow{\mathrm{n}}\right) \frac{e^{i k r^{\prime}+i \overrightarrow{\mathrm{k}} \cdot \overrightarrow{\mathrm{r}}_{i}}}{r^{\prime}}
\end{aligned}
$$

where $\overrightarrow{\mathrm{r}}^{\prime}=\overrightarrow{\mathrm{r}}-\overrightarrow{\mathrm{r}}_{i}$ are the new coordinates and $\overrightarrow{\mathrm{k}}=k \overrightarrow{\mathrm{n}}$. These two equations are the fundamental equations for coherent scattering by a fixed center, which I have discussed in detail in the previous paper. ${ }^{7}$ From the linear properties of both the Schrödinger equation [Eq. (2.5)] and the incident wave in Eq. (2.6), we can express the coherent scattering amplitude as

$$
\begin{aligned}
F_{i}\left(\overrightarrow{\mathrm{k}}_{1}, \overrightarrow{\mathrm{k}}_{2} ; \overrightarrow{\mathrm{n}}\right)= & f\left(\overrightarrow{\mathrm{k}}_{1} ; \overrightarrow{\mathrm{n}}\right) e^{-i\left(\overrightarrow{\mathrm{k}}-\overrightarrow{\mathrm{k}}_{1}\right) \cdot \overrightarrow{\mathrm{r}}_{i}} \\
& +a f\left(\overrightarrow{\mathrm{k}}_{2} ; \overrightarrow{\mathrm{n}}\right) e^{-i\left(\overrightarrow{\mathrm{k}}-\overrightarrow{\mathrm{k}}_{2}\right) \cdot \overrightarrow{\mathrm{r}}_{i}},
\end{aligned}
$$

where $f\left(\overrightarrow{\mathrm{k}}_{j} ; \overrightarrow{\mathrm{n}}\right)$ is the conventional scattering amplitude for an incident beam with momentum $\overrightarrow{\mathrm{k}}_{j}$ scattered by a fixed center with potential $V\left(\overrightarrow{\mathbf{r}}^{\prime}\right)$.

I shall distinguish two different cases in the scattering by multiple centers.

Case I (noninterference scattering). If the centers are far enough apart in comparison with the range of force and the wavelength of incident particles, then each scattering process involves only one of the centers and does not interfere with scattering from the other centers. The observed differential cross section is an independent sum of each individual scattering:

$$
d \Sigma_{I}^{N}\left(\overrightarrow{\mathrm{k}}_{1}, \overrightarrow{\mathrm{k}}_{2} ; \overrightarrow{\mathrm{n}}\right)=\left(1+|a|^{2}\right)^{-1} \sum_{i=1}^{N}\left|F_{i}\left(\overrightarrow{\mathrm{k}}_{1}, \overrightarrow{\mathrm{k}}_{2} ; \overrightarrow{\mathrm{n}}\right)\right|^{2} d \Omega \overrightarrow{\mathrm{n}},
$$

where $d \Omega \overrightarrow{\mathrm{n}}$ is the solid angle. For a single beam, Eq. (2.8) has a simple form, 


$$
\begin{aligned}
d \Sigma_{I}^{N}\left(\overrightarrow{\mathrm{k}}_{1}, \overrightarrow{\mathrm{k}}_{2} ; \overrightarrow{\mathrm{n}}\right) & =\sum_{i=1}^{N}\left|f\left(\overrightarrow{\mathrm{k}}_{1} ; \overrightarrow{\mathrm{n}}\right) e^{-i\left(\overrightarrow{\mathrm{k}}-\overrightarrow{\mathrm{k}}_{1}\right) \cdot \overrightarrow{\mathrm{r}}_{i}}\right|^{2} d \Omega \overrightarrow{\mathrm{n}} \\
& =N\left|f\left(\overrightarrow{\mathrm{k}}_{1} ; \overrightarrow{\mathrm{n}}\right)\right|^{2} d \Omega \overrightarrow{\mathrm{n}} .
\end{aligned}
$$

This is an expected result in conventional scattering. It is stated that the number of scattered particles that emerge per unit time in a small solid angle $d \Omega \vec{n}$ is proportional to the number of independent centers. This type of scattering occurs quite frequently and plays a very important role in atomic, molecular, nuclear, and particle physics.

Case II (interference scattering). If the centers are close together as compared with the wavelength of the incident particles, then the scatterings from individual centers will interfere with each other. This type of scattering occurs in the scattering by crystals. Here the independence of the $N$ scattering centers means that the scattering is weak, and that therefore the wave incident on each center is the original incident wave, and that the waves scattered by other centers may be neglected in comparison with it. Then the total scattering amplitude $F_{C}^{N}\left(\overrightarrow{\mathrm{k}}_{1}, \overrightarrow{\mathrm{k}}_{2} ; \overrightarrow{\mathrm{n}}\right)$ from $N$ centers in the target region is the geometric sum of the individual scattering amplitudes:

$$
F_{C}^{N}\left(\overrightarrow{\mathrm{k}}_{1}, \overrightarrow{\mathrm{k}}_{2} ; \overrightarrow{\mathrm{n}}\right)=\sum_{i=1}^{N} F_{i}\left(\overrightarrow{\mathrm{k}}_{1}, \overrightarrow{\mathrm{k}}_{2} ; \overrightarrow{\mathrm{n}}\right) \text {. }
$$

The experimentally observed differential cross section has the form

$$
d \Sigma_{c}^{N}\left(\overrightarrow{\mathrm{k}}_{1}, \overrightarrow{\mathrm{k}}_{2} ; \overrightarrow{\mathrm{n}}\right)=\left(1+|a|^{2}\right)^{-1}\left|F_{c}^{N}\left(\overrightarrow{\mathrm{k}}_{1}, \overrightarrow{\mathrm{k}}_{2} ; \overrightarrow{\mathrm{n}}\right)\right|^{2} d \Omega \overrightarrow{\mathrm{n}} .
$$

Equations (2.8) and (2.11) form the theoretical basis for the study of the scattering of coherent beams by multiple centers. The observed differential cross sections $d \Sigma_{I}^{N}\left(\overrightarrow{\mathrm{k}}_{1}, \overrightarrow{\mathrm{k}}_{2} ; \overrightarrow{\mathrm{n}}\right)$ and $d \Sigma_{c}^{N}\left(\overrightarrow{\mathrm{k}}_{1}, \overrightarrow{\mathrm{k}}_{2} ; \overrightarrow{\mathrm{n}}\right)$ depend not only on the single-center scattering amplitude, but also on the spatial structure of the target.

\section{PLANE TARGET}

As pointed out in Sec. II, the observed differential cross sections in the scattering of coherent beams depend on the spatial structure of the target. In this section we shall consider a plane target, in which all scattering centers are confined to a flat plane, whose normal is parallel to the momentum difference vector $\vec{k}_{2}-\vec{k}_{1}$. Then

$$
\left(\overrightarrow{\mathrm{k}}_{2}-\overrightarrow{\mathrm{k}}_{1}\right) \cdot \overrightarrow{\mathrm{r}}_{i}=0 \text { for } i=1,2, \ldots, N \text {. }
$$

If the scatterings from these centers are independent and do not interfere with each other, the observed differential cross section is given by Eq. (2.8), and can be written as

$$
\begin{aligned}
d \Sigma_{I}^{N}\left(\overrightarrow{\mathrm{k}}_{1}, \overrightarrow{\mathrm{k}}_{2} ; \overrightarrow{\mathrm{n}}\right)= & \left(1+|a|^{2}\right)^{-1} N \\
& \times\left|f\left(\overrightarrow{\mathrm{k}}_{1} ; \overrightarrow{\mathrm{n}}\right)+a f\left(\overrightarrow{\mathrm{k}}_{2} ; \overrightarrow{\mathrm{n}}\right)\right|^{2} d \Omega \overrightarrow{\mathrm{n}} .
\end{aligned}
$$

In arriving at Eq. (3.2) I have utilized Eqs. (2.7) and (3.1).

If the scatterings from the centers interfere with each other, the observed differential cross section is given by Eq. (2.11), and has the form

$$
\begin{aligned}
d \Sigma_{C}^{N}\left(\overrightarrow{\mathrm{k}}_{1}, \overrightarrow{\mathrm{k}}_{2} ; \overrightarrow{\mathrm{n}}\right)= & \left(1+|a|^{2}\right)^{-1} A^{N} \\
& \times\left|f\left(\overrightarrow{\mathrm{k}}_{1} ; \overrightarrow{\mathrm{n}}\right)+a f\left(\overrightarrow{\mathrm{k}}_{2} ; \overrightarrow{\mathrm{n}}\right)\right|^{2} d \Omega \overrightarrow{\mathrm{n}},
\end{aligned}
$$

where

$$
A^{N}=\left|\sum_{i=1}^{N} e^{-i\left(\overrightarrow{\mathrm{k}}-\overrightarrow{\mathrm{k}}_{1}\right) \cdot \overrightarrow{\mathrm{r}}_{i}}\right|^{2}=\left|\sum_{i=1}^{N} e^{-i\left(\overrightarrow{\mathrm{k}}-\overrightarrow{\mathrm{k}}_{2}\right) \cdot \overrightarrow{\mathrm{r}}_{i}}\right|^{2} .
$$

The factor $A^{N}$ depends on the spatial distribution of centers in the target plane. In arriving at Eq. (3.2) we have also utilized Eqs. (2.7) and (3.1). The differential cross section $d \Sigma_{c}^{N}\left(\overrightarrow{\mathrm{k}}_{1}, \overrightarrow{\mathrm{k}}_{2} ; \overrightarrow{\mathrm{n}}\right)$ in Eq. (3.3) has been factored into two different parts. One of them depends on the spatial structure of the target. The other depends on the single-center scattering amplitude. The differential cross section $d \Sigma_{I}^{N}\left(\overrightarrow{\mathrm{k}}_{1}, \overrightarrow{\mathrm{k}}_{2} ; \overrightarrow{\mathrm{n}}\right)$ in Eq. (3.2) bears the same character. The factorization simplifies the problem. As we shall see in Sec. IV, in the scattering of two coherent beams this factorization is not always possible. On the other hand, for the scattering of a single beam, the factorization is a trivial result, ${ }^{12}$ as we have encountered in atomic, molecular, and nuclear scatterings, and also in crystal diffractions.

If $a=0$ in Eq. (3.3), we obtain the single-beam differential cross section

$$
\left.d \Sigma_{C}^{N}\left(\overrightarrow{\mathrm{k}}_{1}, \overrightarrow{\mathrm{k}}_{2} ; \overrightarrow{\mathrm{n}}\right)\right|_{a=0}=A^{N}\left|f\left(\overrightarrow{\mathrm{k}}_{1} ; \overrightarrow{\mathrm{n}}\right)\right|^{2} d \Omega \overrightarrow{\mathrm{n}}=A^{N} d \sigma\left(\overrightarrow{\mathrm{k}}_{1} ; \overrightarrow{\mathrm{n}}\right),
$$

where $d \sigma\left(\overrightarrow{\mathrm{k}}_{1} ; \overrightarrow{\mathrm{n}}\right)$ is the conventional differential cross section with respect to a single center. Equation (3.5) expresses the fact that the spatial factor $A^{N}$ can be measured through a single-beam scattering experiment. The factor $A^{N}$ might be obtained theoretically, if the spatial distribution of centers in the plane target is known. As an example, we will study the special case in which the centers are randomly distributed. Then the observed structure factor $A^{N}$ given by Eq. (3.4) has to be randomly averaged over these centers. The products of terms pertaining to different centers will vanish on performing a random sum over the positions of these centers, and only the squared modulus of each term remains, so that

$$
A^{N}=N \text {. }
$$

This means that the differential cross section $d \Sigma_{c}^{N}\left(\overrightarrow{\mathrm{k}}_{1}, \overrightarrow{\mathrm{k}}_{2} ; \overrightarrow{\mathrm{n}}\right)$ in Eq. (3.3) for the interference scat- 
tering with respect to randomly distributed centers on a plane is equal to the differential cross section $d \Sigma_{I}^{N}\left(\overrightarrow{\mathrm{k}}_{1}, \overrightarrow{\mathrm{k}}_{2} ; \overrightarrow{\mathrm{n}}\right)$ in Eq. (3.2) for the noninterference scattering whether the centers are distributed in an ordered or a random way on a plane.

As a result of this factorization, the scattering of two coherent beams by multiple centers confined in a plane becomes a rather simple case. The experiment will lead to the direct determination of the real and imaginary parts of the scattering amplitude at all angles. The analysis of such a determination is the same as in the case of a single center, which has been considered previously. ${ }^{7}$ I shall not repeat such a discussion here.

\section{NONINTERFERENCE SCATTERING}

In this section I make a detailed study of the noninterference scattering as outlined in case I of Sec. II. For the sake of simplicity, I assume that the scattering centers are not only far apart, but also randomly distributed in the three-dimensional target region. This type of scattering with a single beam is very common, and has been extensively used in the experimental study of microscopic objects. The experimentally measured differential cross sections from the single-beam scatterings have provided us with ample knowledge of the magnitudes of the scattering amplitude in the case where the scattering center is a single atom, molecule, or nucleus. Through the proposed scattering by two coherent beams, we wish to recover the missing information on the phase of the scattering amplitude.

For a single scattering center, the measured differential and total cross sections from two coherent beams provide, as I have shown, ${ }^{7}$ direct information on the phase of the scattering amplitude. For multiple scattering centers, the problem is not that simple. We shall see that the directly measured differential and total cross sections do not provide any information on the phase of the scattering amplitude. The reason is not difficult to explain. Using Eq. (2.7), the noninterference differential cross section in Eq. (2.8) can be rewritten as

$$
\begin{aligned}
d \Sigma_{I}^{N}\left(\overrightarrow{\mathrm{k}}_{1}, \overrightarrow{\mathrm{k}}_{2} ; \overrightarrow{\mathrm{n}}\right) & =\left(1+|a|^{2}\right)^{-1} \sum_{i=1}^{N}\left\{\left|f\left(\overrightarrow{\mathrm{k}}_{1} ; \overrightarrow{\mathrm{n}}\right)\right|^{2}+|a|^{2}\left|f\left(\overrightarrow{\mathrm{k}}_{2} ; \overrightarrow{\mathrm{n}}\right)\right|^{2}+2 \operatorname{Re}\left[a f\left(\overrightarrow{\mathrm{k}}_{2} ; \overrightarrow{\mathrm{n}}\right) f^{*}\left(\overrightarrow{\mathrm{k}}_{1} ; \overrightarrow{\mathrm{n}}\right) e^{\left.\left.-i\left(\overrightarrow{\mathrm{k}}_{1}-\overrightarrow{\mathrm{k}}_{2}\right) \cdot \overrightarrow{\mathrm{r}}_{i}\right]\right\} d \Omega \overrightarrow{\mathrm{n}}}\right.\right. \\
& =\left(1+|a|^{2}\right)^{-1} N\left\{\left|f\left(\overrightarrow{\mathrm{k}}_{1} ; \overrightarrow{\mathrm{n}}\right)\right|^{2}+|a|^{2}\left|f\left(\overrightarrow{\mathrm{k}}_{2} ; \overrightarrow{\mathrm{n}}\right)\right|^{2}\right\} d \Omega \overrightarrow{\mathrm{n}} .
\end{aligned}
$$

As a result of the random summation, the cross term in Eq. (4.1) vanishes. The differential cross section $d \Sigma_{I}^{N}\left(\overrightarrow{\mathrm{k}}_{1}, \overrightarrow{\mathrm{k}}_{2} ; \overrightarrow{\mathrm{n}}\right)$ depends only on the magnitude of the scattering amplitude $f\left(\overrightarrow{\mathrm{k}}_{1} ; \overrightarrow{\mathrm{n}}\right)$. Equation (4.1) can also be obtained for two incoherent beams. Hence the randomness of the scattering centers has eliminated the coherence effect and dropped out the phase information in the measured differential cross section $d \Sigma_{I}^{N}\left(\overrightarrow{\mathrm{k}}_{1}, \overrightarrow{\mathrm{k}}_{2} ; \overrightarrow{\mathrm{n}}\right)$.

To recover the lost information one has to employ the coincidence measurement. The coincidence measurement, which was first introduced by Dunworth, ${ }^{13}$ has been utilized very extensively as a method for measuring various properties of nuclear energy levels. ${ }^{10}$ There are a number of different types of coincidence experiments. Here we shall only refer to the simplest one, which consists of the coincidence measurement of the differential cross section at two distinct directions. For a single scattering center, the coincidence measurement leads to the observed quantity ${ }^{10}$

$$
\begin{aligned}
& K_{i}\left(\overrightarrow{\mathrm{k}}_{1}, \overrightarrow{\mathrm{k}}_{2} ; \overrightarrow{\mathrm{n}}, \overrightarrow{\mathrm{n}}^{\prime}\right) \\
& \quad \equiv\left(1+|a|^{2}\right)^{-2}\left|F_{i}\left(\overrightarrow{\mathrm{k}}_{1}, \overrightarrow{\mathrm{k}}_{2} ; \overrightarrow{\mathrm{n}}\right)\right|^{2}\left|F_{i}\left(\overrightarrow{\mathrm{k}}_{1}, \overrightarrow{\mathrm{k}}_{2} ; \overrightarrow{\mathrm{n}}^{\prime}\right)\right|^{2},
\end{aligned}
$$

which depends on both the directions $\vec{n}$ and $\vec{n}^{\prime}$. For the noninterference scatterings by $N$ independent centers, the observed quantity in the coincidence measurement is the direct sum of these quantities from each individual center:

$$
K_{I}^{N}\left(\overrightarrow{\mathrm{k}}_{1}, \overrightarrow{\mathrm{k}}_{2} ; \overrightarrow{\mathrm{n}}, \overrightarrow{\mathrm{n}}^{\prime}\right)=\sum_{i=1}^{N} K_{i}\left(\overrightarrow{\mathrm{k}}_{1}, \overrightarrow{\mathrm{k}}_{2} ; \overrightarrow{\mathrm{n}}, \overrightarrow{\mathrm{n}}^{\prime}\right) .
$$

Using Eqs. (2.7) and (4.2), we can rewrite the above equation as

$$
\begin{aligned}
K_{I}^{N}\left(\overrightarrow{\mathrm{k}}_{1}, \overrightarrow{\mathrm{k}}_{2} ; \overrightarrow{\mathrm{n}}, \overrightarrow{\mathrm{n}}^{\prime}\right)=\left(1+|a|^{2}\right)^{-2} \sum_{i=1}^{N} & \left\{\left[\left|f\left(\overrightarrow{\mathrm{k}}_{1} ; \overrightarrow{\mathrm{n}}\right)\right|^{2}+\left|a f\left(\overrightarrow{\mathrm{k}}_{2} ; \overrightarrow{\mathrm{n}}\right)\right|^{2}\right]\left[\left|f\left(\overrightarrow{\mathrm{k}}_{1} ; \overrightarrow{\mathrm{n}}^{\prime}\right)\right|^{2}+\left|a f\left(\overrightarrow{\mathrm{k}}_{2} ; \overrightarrow{\mathrm{n}}^{\prime}\right)\right|^{2}\right]\right. \\
& +2|a|^{2} \operatorname{Re}\left[f\left(\overrightarrow{\mathrm{k}}_{2} ; \overrightarrow{\mathrm{n}}\right) f^{*}\left(\overrightarrow{\mathrm{k}}_{1} ; \overrightarrow{\mathrm{n}}\right) f^{*}\left(\overrightarrow{\mathrm{k}}_{2} ; \overrightarrow{\mathrm{n}}^{\prime}\right) f\left(\overrightarrow{\mathrm{k}}_{1} ; \overrightarrow{\mathrm{n}}^{\prime}\right)\right] \\
& +2 \operatorname{Re}\left[a^{2} f\left(\overrightarrow{\mathrm{k}}_{2} ; \overrightarrow{\mathrm{n}}\right) f^{*}\left(\overrightarrow{\mathrm{k}}_{1} ; \overrightarrow{\mathrm{n}}\right) f\left(\overrightarrow{\mathrm{k}}_{2} ; \overrightarrow{\mathrm{n}}^{\prime}\right) f^{*}\left(\overrightarrow{\mathrm{k}}_{1} ; \overrightarrow{\mathrm{n}}^{\prime}\right) e^{\left.-2 i\left(\overrightarrow{\mathrm{k}}_{1}-\overrightarrow{\mathrm{k}}_{2}\right) \cdot \overrightarrow{\mathrm{r}}_{i}\right]}\right. \\
& +2\left[\left|f\left(\overrightarrow{\mathrm{k}}_{1} ; \overrightarrow{\mathrm{n}}\right)\right|^{2}+\left|a f\left(\overrightarrow{\mathrm{k}}_{2} ; \overrightarrow{\mathrm{n}}\right)\right|^{2}\right] \operatorname{Re}\left[a f\left(\overrightarrow{\mathrm{k}}_{2} ; \overrightarrow{\mathrm{n}}^{\prime}\right) f^{*}\left(\overrightarrow{\mathrm{k}}_{1} ; \overrightarrow{\mathrm{n}}^{\prime}\right) e^{\left.-i\left(\overrightarrow{\mathrm{k}}_{1}-\overrightarrow{\mathrm{k}}_{2}\right) \cdot \overrightarrow{\mathrm{r}}_{i}\right]}\right. \\
& +2\left[\left|f\left(\overrightarrow{\mathrm{k}}_{1} ; \overrightarrow{\mathrm{n}}^{\prime}\right)\right|^{2}+\left|a f\left(\overrightarrow{\mathrm{k}}_{2} ; \overrightarrow{\mathrm{n}}^{\prime}\right)\right|^{2}\right] \operatorname{Re}\left[a f\left(\overrightarrow{\mathrm{k}}_{2} ; \overrightarrow{\mathrm{n}}\right) f^{*}\left(\overrightarrow{\mathrm{k}}_{1} ; \overrightarrow{\mathrm{n}}\right) e^{\left.\left.-i\left(\overrightarrow{\mathrm{k}}_{1}-\overrightarrow{\mathrm{k}}_{2}\right) \cdot \overrightarrow{\mathrm{r}}_{i}\right]\right\} .}\right.
\end{aligned}
$$


After performing a random summation over the scattering centers, one arrives at the expression

$$
\begin{aligned}
& K_{I}^{N}\left(\overrightarrow{\mathrm{k}}_{1}, \overrightarrow{\mathrm{k}}_{2} ; \overrightarrow{\mathrm{n}}, \overrightarrow{\mathrm{n}}^{\prime}\right)=\left(1+|a|^{2}\right)^{-2} N\{\left\{\left|f\left(\overrightarrow{\mathrm{k}}_{1} ; \overrightarrow{\mathrm{n}}\right)\right|^{2}+\left|a f\left(\overrightarrow{\mathrm{k}}_{2} ; \overrightarrow{\mathrm{n}}\right)\right|^{2}\right]\left[\left|f\left(\overrightarrow{\mathrm{k}}_{1} ; \overrightarrow{\mathrm{n}}^{\prime}\right)\right|^{2}+\left|a f\left(\overrightarrow{\mathrm{k}}_{2} ; \overrightarrow{\mathrm{n}}^{\prime}\right)\right|^{2}\right] \\
&\left.+2|a|^{2} \operatorname{Re}\left[f\left(\overrightarrow{\mathrm{k}}_{2} ; \overrightarrow{\mathrm{n}}\right) f^{*}\left(\overrightarrow{\mathrm{k}}_{1} ; \overrightarrow{\mathrm{n}}\right) f^{*}\left(\overrightarrow{\mathrm{k}}_{2} ; \overrightarrow{\mathrm{n}}^{\prime}\right) f\left(\overrightarrow{\mathrm{k}}_{1} ; \overrightarrow{\mathrm{n}}^{\prime}\right)\right]\right\} .
\end{aligned}
$$

For elastic scattering the unitarity relation ${ }^{12}$ can be expressed as

$$
f\left(\overrightarrow{\mathrm{k}}_{1} ; \overrightarrow{\mathrm{n}}_{2}\right)-f^{*}\left(\overrightarrow{\mathrm{k}}_{2} ; \overrightarrow{\mathrm{n}}_{1}\right)=\frac{i k}{2 \pi} \int f\left(\overrightarrow{\mathrm{k}}_{1} ; \overrightarrow{\mathrm{n}}\right) f^{*}\left(\overrightarrow{\mathrm{k}}_{2} ; \overrightarrow{\mathrm{n}}\right) d \Omega \overrightarrow{\mathrm{n}}
$$

With the help of the above relation, one can perform the integration of the measured quantity $K_{I}^{N}\left(\overrightarrow{\mathrm{k}}_{1}, \overrightarrow{\mathrm{k}}_{2} ; \overrightarrow{\mathrm{n}}, \overrightarrow{\mathrm{n}}^{\prime}\right)$ with respect to $d \Omega_{\overrightarrow{\mathrm{n}}}$ and $d \Omega_{\overrightarrow{\mathrm{n}}^{\prime}}$ :

$$
\begin{aligned}
\sum_{I}^{C}\left(\overrightarrow{\mathrm{k}}_{1}, \overrightarrow{\mathrm{k}}_{2}\right) \equiv & \int K_{I}^{N}\left(\overrightarrow{\mathrm{k}}_{1}, \overrightarrow{\mathrm{k}}_{2} ; \overrightarrow{\mathrm{n}}, \overrightarrow{\mathrm{n}}^{\prime}\right) d \Omega_{\overrightarrow{\mathrm{n}}} d \Omega_{\overrightarrow{\mathrm{n}}^{\prime}} \\
= & N \sigma^{2}(k)+\frac{8 \pi^{2} N|a|^{2}}{k^{2}\left(1+|a|^{2}\right)^{2}} \\
& \times\left|f\left(\overrightarrow{\mathrm{k}}_{1} ; \overrightarrow{\mathrm{n}}_{2}\right)-f^{*}\left(\overrightarrow{\mathrm{k}}_{2} ; \overrightarrow{\mathrm{n}}_{1}\right)\right|^{2},
\end{aligned}
$$

where

$$
\sigma(k)=\sigma\left(\overrightarrow{\mathrm{k}}_{i}\right)=\int\left|f\left(\overrightarrow{\mathrm{k}}_{i} ; \overrightarrow{\mathrm{n}}\right)\right|^{2} d \Omega \overrightarrow{\mathrm{n}} .
$$

is the conventional total cross section. The symmetry with respect to time reversal ${ }^{12}$ yields the following restriction on the scattering amplitude:

$$
f\left(\overrightarrow{\mathrm{k}}_{1} ; \overrightarrow{\mathrm{n}}_{2}\right)=f\left(-\overrightarrow{\mathrm{k}}_{2},-\overrightarrow{\mathrm{n}}_{1}\right) \text {. }
$$

If the potential $V(\overrightarrow{\mathrm{r}})$ is invariant under space reflection, ${ }^{12}$ then

$$
f\left(\overrightarrow{\mathrm{k}}_{1} ; \overrightarrow{\mathrm{n}}_{2}\right)=f\left(-\overrightarrow{\mathrm{k}}_{1} ;-\overrightarrow{\mathrm{n}}_{2}\right) .
$$

From Eqs. (4.7), (4.9), and (4.10), we might express the imaginary part of the scattering amplitude in terms of measured quantities, which can be obtained through the scattering experiment with $N$ independent scattering centers:

$$
\operatorname{Im} f\left(\overrightarrow{\mathrm{k}}_{1} ; \overrightarrow{\mathrm{n}}\right)=\frac{k}{4 \pi} \frac{1+|a|^{2}}{\sqrt{2}|a|}\left[N^{-1} \Sigma_{I}^{c}\left(\overrightarrow{\mathrm{k}}_{1}, \overrightarrow{\mathrm{k}}_{2}\right)-\sigma^{2}(k)\right]^{\mathrm{i} / 2} \text {. }
$$

Here I would like to say a few words about the noninterference scattering by centers which form an ideal crystal. This type of scattering depends on the directional difference $\left(\vec{k}_{1}-\vec{k}_{2}\right)$ of two coherent beams. If the difference $\left(\vec{k}_{1}-\vec{k}_{2}\right)$ is not equal to a reciprocal lattice vector of the crystal, then the noninterference scattering by these centers is essentially equivalent to that by randomly dis tributed centers. If the difference $\left(\vec{k}_{1}-\vec{k}_{2}\right)$ is equal to a reciprocal-lattice vector of the crystal, then the noninterference differential cross section in Eq. (2.8) can be rewritten as

$$
\begin{aligned}
d \Sigma_{I}^{N}\left(\overrightarrow{\mathrm{k}}_{1}, \overrightarrow{\mathrm{k}}_{2} ; \overrightarrow{\mathrm{n}}\right) & =\left(1+|a|^{2}\right)^{-1} \sum_{i=1}^{N}\left\{\left|f\left(\overrightarrow{\mathrm{k}}_{1} ; \overrightarrow{\mathrm{n}}\right)\right|^{2}+|a|^{2}\left|f\left(\overrightarrow{\mathrm{k}}_{2} ; \overrightarrow{\mathrm{n}}\right)\right|^{2}+2 \operatorname{Re}\left[a f\left(\overrightarrow{\mathrm{k}}_{2} ; \overrightarrow{\mathrm{n}}\right) f^{*}\left(\overrightarrow{\mathrm{k}}_{1} ; \overrightarrow{\mathrm{n}}\right) e^{\left.-i\left(\overrightarrow{\mathrm{k}}_{1}-\overrightarrow{\mathrm{k}}_{2}\right) \cdot \overrightarrow{\mathrm{r}}_{i}\right]}\right\} d \Omega_{\overrightarrow{\mathrm{n}}}\right. \\
& =\left(1+|a|^{2}\right)^{-1} N\left\{\left|f\left(\overrightarrow{\mathrm{k}}_{1} ; \overrightarrow{\mathrm{n}}\right)\right|^{2}+|a|^{2}\left|f\left(\overrightarrow{\mathrm{k}}_{2} ; \overrightarrow{\mathrm{n}}\right)\right|^{2}+2 \operatorname{Re}\left[a f\left(\overrightarrow{\mathrm{k}}_{2} ; \overrightarrow{\mathrm{n}}\right) f^{*}\left(\overrightarrow{\mathrm{k}}_{1} ; \overrightarrow{\mathrm{n}}\right)\right]\right\} d \Omega_{\overrightarrow{\mathrm{n}}} .
\end{aligned}
$$

The analysis of such a determination is rather a simple one, which I have discussed in the scattering by a single center previously. ${ }^{7}$ These above results are quite easy to obtain. I leave it to the interested readers.

\section{INTERFERENCE SCATTERING}

In this section we assume that the scattering centers are distributed in three-dimensional space in an orderly way. That is, these centers form an ideal crystal. The subject under discussion is actually a diffraction of two coherent beams by a crystal.

The position vector of each center is now expressible in terms of three fundamental lattice vectors $\vec{a}, \vec{b}$, and $\vec{c}$ :

$$
\overrightarrow{\mathrm{r}}_{i}=\alpha_{i} \overrightarrow{\mathrm{a}}+\beta_{i} \overrightarrow{\mathrm{b}}+\gamma_{i} \overrightarrow{\mathrm{c}}
$$

where $\alpha_{i}, \beta_{i}$, and $\gamma_{i}$ are arbitrary integers. From Eqs. (2.7) and (2.10), the scattering amplitude of the crystal diffraction has the form

$$
\begin{aligned}
& F_{C}^{N}\left(\overrightarrow{\mathrm{k}}_{1}, \overrightarrow{\mathrm{k}}_{2} ; \overrightarrow{\mathrm{n}}\right) \\
& \quad=\sum_{i=1}^{N} e^{-i\left(\overrightarrow{\mathrm{k}}-\overrightarrow{\mathrm{k}}_{1}\right) \cdot \overrightarrow{\mathrm{r}}_{i}}\left[f\left(\overrightarrow{\mathrm{k}}_{1} ; \overrightarrow{\mathrm{n}}\right)+a f\left(\overrightarrow{\mathrm{k}}_{2} ; \overrightarrow{\mathrm{n}}\right) e^{\left.-i\left(\overrightarrow{\mathrm{k}}_{1}-\overrightarrow{\mathrm{k}}_{2}\right) \cdot \overrightarrow{\mathrm{r}}_{i}\right]}\right.
\end{aligned}
$$

In most cases the number $N$ of scattering centers is usually large. The summation in Eq. (5.2) is characteristically dependent on the directional difference $\left(\vec{k}_{1}-\vec{k}_{2}\right)$ of two coherent beams. If

$$
\overrightarrow{\mathrm{k}}_{1}-\overrightarrow{\mathrm{k}}_{2} \neq \overrightarrow{\mathrm{K}} \text {, }
$$

where $\vec{K}$ is a reciprocal-lattice vector of the crystal, then the diffractions from the two coherent beams are incoherent and the resultant diffrac- 
tion would seem to come from two incoherent beams. This is certainly an uninteresting case. If

$$
\overrightarrow{\mathrm{k}}_{1}-\overrightarrow{\mathrm{k}}_{2}=\overrightarrow{\mathrm{K}}
$$

then the diffractions from the two coherent beams are coherent and the resultant amplitude has the form

$$
F_{C}^{N}\left(\overrightarrow{\mathrm{k}}_{1}, \overrightarrow{\mathrm{k}}_{2} ; \overrightarrow{\mathrm{n}}\right)=\sum_{i=1}^{N} e^{-i\left(\overrightarrow{\mathrm{k}}-\overrightarrow{\mathrm{k}}_{1}\right) \cdot \overrightarrow{\mathrm{r}}_{i}}\left[f\left(\overrightarrow{\mathrm{k}}_{1} ; \overrightarrow{\mathrm{n}}\right)+a f\left(\overrightarrow{\mathrm{k}}_{2} ; \overrightarrow{\mathrm{n}}\right)\right]
$$

The experimentally measured differential cross section in Eq. (2.11) can be expressed as

$$
d \Sigma_{C}^{N}\left(\overrightarrow{\mathrm{k}}_{1}, \overrightarrow{\mathrm{k}}_{2} ; \overrightarrow{\mathrm{n}}\right)=\left(1+|a|^{2}\right)^{-1}|E|^{2}|G|^{2} d \Omega \overrightarrow{\mathrm{n}},
$$

where

$$
E=f\left(\overrightarrow{\mathrm{k}}_{1} ; \overrightarrow{\mathrm{n}}\right)+a f\left(\overrightarrow{\mathrm{k}}_{2} ; \overrightarrow{\mathrm{n}}\right)
$$

and

$$
G=\sum_{i=1}^{N} e^{-i\left(\overrightarrow{\mathrm{k}}-\overrightarrow{\mathrm{k}}_{1}\right) \cdot \overrightarrow{\mathrm{r}}_{i}}=\sum_{i=1}^{N} e^{-i\left(\overrightarrow{\mathrm{k}}-\overrightarrow{\mathrm{k}}_{2}\right) \cdot \overrightarrow{\mathrm{r}}_{i}} .
$$

In the theory of crystal diffraction, $E$ is called the structure factor and $G$ the lattice factor, which depends on the type of the lattice. The factor $G$ can be determined and investigated by the singlebeam diff raction method. It is presumably the main topic in the experimental study of crystal diffractions. The diffraction of two coherent beams does not provide any new information on this factor. The structure factor $E$ describes the relative intensities of the various crystal reflections and depends on the contents of the unit cell, that is, on the number, type, and distribution of atoms in the cell. In other words, the factor $E$ is the scattering amplitude from all atoms in the unit cell. In our case the unit cell is formed by one single scattering center and the factor $E$, as expressed in Eq. (5.7), is the scattering amplitude of two coherent beams by a single center. The diffraction by two coherent beams does add new information on the structure factor $E$. From the diffraction of a single beam, one only determines the magnitude of the structure factor, which is $f\left(\overrightarrow{\mathrm{k}}_{1} ; \overrightarrow{\mathrm{n}}\right)$ in this case. Through the interference between the diffractions from two coherent beams, the phase of the structure factor can also be determined.

In crystal diffraction, one is more interested in the lattice factor $G$ than the structure factor $E$. It is stated in most textbooks ${ }^{14}$ that the structure factor is determined from atomic theory. Often the Born amplitude is quoted as the expression for the structure factor. This amplitude contains some truth, but is still far from being a true structure factor, namely, the scattering amplitude for all atoms in the unit cell. It is known that not any function can be the scattering amplitude. In order to be a scattering amplitude it must satisfy the unitarity relation. The quoted Born amplitude ${ }^{14}$ is often a real quantity, which can never satisfy the unitarity relation. There are many elaborate theories in atomic physics, but an atomic scattering amplitude, which is free from any approximation and assumption, is still unknown. The experimentally and completely measured structure factor will be helpful to build a more complete atomic theory.

The crystal diffraction by two coherent beams is not only feasible theoretically, but is also realizable experimentally. To be more specific, the crystal diffraction by two coherent beams was carried out by Marton, Simpson, and Suddeth ${ }^{11}$ more than 20 years ago. The main motivation for their experiment was to show directly the interference effect of two coherent electron beams at an energy of $60 \mathrm{keV}$. The interference fringes are very luminous, show strong contrast, and are steady. The data were actually overlooked and were never analyzed. Twenty years is rather a long time within the progress of modern physics. There have been some important developments in coherent electron beams. With the field-emission method, ${ }^{15}$ this experiment can be performed more easily. ${ }^{16}$

Note added in manuscript. I have learned recently that experiments ${ }^{17}$ have also been done on the interference effect of two coherent neutron beams at wavelengths of about $4 \AA$.

\section{ACKNOWLEDGMENTS}

The author is very grateful to Professor J. A. Simpson, Professor A. V. Crewe, Dr. M. Isaacson, Professor T. Welton, and their groups for the interesting discussions on the experimental aspects of the scattering of coherent electron beams. To his colleagues, Professor D. Kaplan and Professor D. Long, go thanks for many stimulating conversations on matters presented here.
*Work supported in part by the Research Division of Virginia Polytechnic Institute and State University, Blacksburg, Virginia 24061.

${ }^{1}$ G. Breit, E. U. Condon, and R. D. Present, Phys. Rev.
50, 825 (1936); J. D. Jackson and J. M. Blatt, Rev. Mod. Phys. 22 , 77 (1950); H. A. Bethe, Phys. Rev. $\underline{76}$, 161 (1949); Ann. Phys. (N.Y.) 3, 190 (1958).

${ }^{2}$ R. A. Arndt, R. H. Hackman, and L. D. Roper, Phys. 
Rev. C $\underline{9}, 555$ (1974); M. H. MacGregor, R. A. Arndt, and R. $\vec{M}$. Wright, Phys. Rev. 182, 1714 (1969).

${ }^{3}$ M. H. MacGregor, M. J. Moravsik, and H. P. Stapp, Ann. Rev. Nucl. Sci. 10, 291 (1960); M. H. MacGregor, R. A. Arndt, and A. A. Dubow, Phys. Rev. 135, B628 (1964); L. D. Roper, R. M. Wright, and B. T. Feld, Phys. Rev. 138, B190 (1965).

${ }^{4}$ L. D. Roper and D. S. Bailey, Phys. Rev. 155, 1744 (1967); J. H. Crichton, Nuovo Cimento 45, 256 (1966).

${ }^{5}$ R. Hanbury-Brown and R. Q. Twiss, Philos. Mag. 45, 663 (1954); Proc. R. Soc. A 242, 300 (1957); 243, 291 (1957); E. M. Purcell, Nature 178, 1449 (1956); M. L. Goldberger, H. W. Lewis, and K. M. Watson, Phys. Rev. 132, 2764 (1963); R. G. Newton, Scattering Theory of Waves and Particles (McGraw-Hill, New York, 1966).

${ }^{6}$ E. P. Wigner, in Twelfth Eastern Theoretical Physics Conference, Blacksburg, Va., 1973 (unpublished). ${ }^{7}$ Ming Chiang Li, Phys. Rev. A 9, 1635 (1974).

${ }^{8}$ L. Marton, Phys. Rev. 85, 1057 (1952); L. Marton, J. A. Simpson, and J. A. Suddeth, Phys. Rev. 90 , 490 (1953); L. Marton, Science 118, 470 (1953);
G. Mollenstedt and H. Duker, Z. Naturforsch. $\underline{8}, 79$ (1953); Naturwissenschaften 42,41 (1954); H. Duker, Z. Naturforsch. 10, 256 (1955).

${ }^{9}$ J. A. Simpson (private communication).

${ }^{10}$ L. C. Biedenharn and M. E. Rose, Rev. Mod. Phys. 25, 729 (1953); L. C. Biedenharn, Nuclear Spectroscopy, edited by F. Ajzenberg-Selove (Academic, New York, 1960).

${ }^{11}$ L. Marton, J. A. Simpson, and J. A. Suddeth, Rev. Sci. Instrum. 25, 1099 (1954).

${ }^{12}$ L. D. Landau and E. M. Lifshitz, Quantum Mechanics, (Pergamon, New York, 1965).

${ }^{13}$ J. W. Dunworth, Rev. Sci. Instrum. 11, 167 (1940).

${ }^{14}$ For example, R. E. Peierls, Quantum Theory of Solids (Oxford U. P., London, 1955); T. B. Rymer, Electron Diffraction (Methuen, London, 1970).

${ }^{15} \mathrm{~J}$. W. Gadzuk and E. W. Plummer, Rev. Mod. Phys. 45,487 (1973).

${ }^{16}$ G. Saxon, Optik 35, 195 (1972).

${ }^{17} \mathrm{H}$. Maier-Leibnitz and T. Springer, Z. Phys. 167, 386 (1962); F. J. Landkammer, Z. Phys. 189, 113 (1966); P. Korpiun, Z. Phys. 195, 146 (1966). 